Breve historia de un (des)entendimiento: la ciencia y la literatura en el devenir...

\title{
BREVE HISTORIA DE UN (DES)ENTENDIMIENTO: LA CIENCIA Y LA LITERATURA EN EL DEVENIR DE LA MODERNIDAD*
}

\author{
Maria Helena SANTANA \\ Universidade de Coimbra \\ Centro de Literatura Portuguesa \\ mahesa@ fl.uc.pt
}

1 contrario de lo que se pudiera pensar — tantos son los lugares de encuentro entre el
imaginario artístico y el científico a lo largo del tiempo- las relaciones entre Literatura y
Ciencia nunca han sido de simple intercambio. Como sistemas de representación del mundo con ambición de universalidad, inevitavelmente se han constituido como «narrativas rivales», o sea, diferentes formas de interpretar lo real, con destino divergente anunciado. La armonía, si alguna vez ha llegado a existir, dejó de ser posible en la Modernidad (a lo largo de los dos últimos siglos, grosso modo), a partir del momento en que las dos instituciones adquirieron conciencia de su poder simbólico $\mathrm{y}$, principalmente, de su desigual influencia en la esfera sociocultural.

Vivimos en una era científica, y sería fútil negar esta evidencia. Paso a paso la Ciencia ha ido imponiendo sus modelos heurísticos y frecuentemente teleológicos ${ }^{1}$, antes compartidos ampliamente por las religiones y las Humanidades. En proceso de autonomización, la creación literaria se confinó gradualmente al espacio artístico a medida en que fue perdiendo expresión en el espacio público. Sabiéndose incapaz de dialogar con las ciencias en el plano del conocimiento objetivo, de la misma manera que dejó de competir con la política, la economia o los media en el plano pragmático, la Literatura siguió resistiendo (en palabras de Jacques Dubois), como «reserva» o «suplemento» cognitivo: «En fin de compte, philosophie, science ou politique n'agissent jamais qu'obliquement sur les pratiques littéraires. Inversement, par rapport à elles, la littérature se tient en position de "réserve", de suppléance» (Dubois, 1986: 60).

\footnotetext{
* Una versión portuguesa de este artículo ha sido publicada en Biblos. Rev. da Fac. de Letras. Universidade de Coimbra. n. s. VI Ciência n(e)as Artes (2008), pp. 17-21. Se agradece a Eloísa Álvarez la traducción al castellano.

${ }^{1}$ Acerca de la construcción y sobredeterminación cultural de la idea de Ciencia cf. Mary Midgley, Science as Salvation. A Modern Myth and Its Meaning : «The promise of satisfying spiritual needs has played a great part in establishing the special glory of the abstraction "science" in our culture. [...] It has built up a strong emotive and romantic conception of "science' as a spiritual power, a most ambitious estimate of what this abstraction is and can do» (Midgley, 1992: 2).
} 


\section{Maria Helena Santana}

Y en esa cualidad de resistente, desventajosa, es como tiene sentido confrontar Literatura y Ciencia en una mirada retrospectiva, a partir de la actualidad. Voy a centrar mi lectura en tres periodos de la historia literaria reciente: el Realismo-Naturalismo, el Modernismo y el Pos-Modernismo, por ser momentos particularmente reveladores de la escisión entre las «dos culturas»: periodos en que la Ciencia deja de ser entendida como un simple tema literario para constituirse en objeto central de una tensión más profunda y perturbadora.

1. No podemos ignorar en esta reflexión inicial la historicidad de los conceptos de Ciencia y de Literatura, que se especifican sólo paulatinamente, sobre todo después de la Ilustración. Hasta comienzos del XIX, como sabemos, la idea de Literatura no existía en los términos en que hoy la concebimos — como categoría de la expresión artística一, acogiendo en un mismo lexema saberes y discursos mucho más amplios que los incluidos en las llamadas «Belles Lettres» (Silva, 1984: 9-13). La Ciencia, a su vez, como término y como concepto disfrutaba de idéntica amplitud semántica, a pesar de existir una tradición diferenciadora de las ramas epistemológicas que la constituían, tal y como sucedía, además, con la mayor parte de los géneros literarios. En contrapartida, la fractura entre Letras y Ciencias era ya, a pesar de las ambiguedades citadas, bastante más evidente de la que hoy se observa: un «hombre de letras», expresión popularizada en el XIX, cubría actividades asociadas al uso retórico (especulativo, pedagógico, creativo...) de la palabra; incluía al historiador y al filósofo, al poeta, al parlamentario o al periodista, pero no se confundía con el «científico», expresión reservada a áreas teórico-experimentales del saber ${ }^{2}$. El eclecticismo del «hombre de letras» reforzaba así la afinidad entre los dominios humanísticos, en un momento en que las ciencias se organizaban ya en diferentes sectores de especialización.

En una primera fase, la Literatura sacó partido de la indiferenciación de las Humanidades: al mismo tiempo que legitimaba su espacio, prestigiándose como arte, sus áreas de irradiación social se mantenían bastante permeables y accesibles. En un contexto favorable al poder de la imprenta, el escritor (el poeta romántico en particular) empezó a disfrutar de un reconocimiento público notable, que le permitió en muchos casos erigirse en voz colectiva de una nación: véase el ejemplo de Victor Hugo, paradigma europeo del poeta-profeta ${ }^{3}$, o, en Portugal, el de Garrett y Herculano.

La ola de novela de costumbres, todavía en tiempo romántico, trajo un nuevo fulgor a la influencia social del escritor. La sociedad burguesa, en rápida y profunda transformación, requería un arte literario capaz de representar las tensiones emergentes en su realidad empírica. Balzac, en particular, tuvo esa memorable intuición, justamente cuando comenzaba a agotarse el aura romántica de la poesía. Bajo el estatuto aparentemente humilde de «secretario» de la sociedad, le abrió camino a una fecunda derivación de la escritura literaria; durante varias décadas, la novela de costumbres le permitiría al escritor trabajar en un vacío cognitivo que la sociología todavía no había llegado a ocupar.

\footnotetext{
${ }^{2}$ Sobre estas categorias cf. Charle (1990); Polanco (1989).

${ }^{3}$ Sobre este concepto véase Paul Bénichou (1977).
} 
Sin embargo, ese momento crítico no tardaría en llegar. A mediados del siglo XIX empezaba a difundirse, a partir sobre todo de Francia, el Positivismo, que proclamaba la sistematización de los saberes bajo la idea unificadora del progreso social. En ese mismo contexto empiezan también a formarse las llamadas «ciencias humanas» — como la Sociología (también designada Mesología), la Antropología o (más tarde) la Psicología- que prometían describir por métodos racionales las mismas realidades que la Filosofía describía de forma especulativa y la Literatura de forma intuitiva: concretamente, las materias relacionadas con el funcionamiento de las relaciones interpersonales o los fenómenos del psiquismo humano. Nacía así un nuevo campo epistemológico (con su vanguardia en la Sociología ${ }^{4}$ ) que, por la afirmación añadida del «valor» científico, tenía legitimidad para reivindicar un espacio de conocimiento que los mismos literatos consideraban estructurante; un saber que tradicionalmente se asociaba a la Literatura, pero fundamentado en presupuestos de verdad y de objectividad.

Cuando las señales de esta irónica «competencia» se hicieron perceptibles no hubo, ni podía haber, más que una inquietud latente, traducida de forma contradictoria en los medios literarios: o reactiva (como más adelante veremos) o promisora, a veces incluso eufórica. La estrategia de los novelistas, consistió, comprensiblemente, en aliarse a las ciencias que nacían, y cuyo magisterio insistían en subrayar. Recordemos, por ejemplo, la famosa intervención de Eça de Queirós en las Conferencias del Casino lisboeta, en 1871, en que se defendió la subordinación de lo «bello» artístico a la «ley moral y científica»; por lo demás, esas Conferencias pretendían dar testimonio en Portugal del nuevo espíritu científico interdisciplinar. En el mismo sentido se orientan las revistas literarias de esta época, que empezaron a incluir muchos artículos de divulgación científica positivista, enriquecidos con pomposas citas de ideólogos como Taine, Proudhon, Comte, Spencer, Stuart Mill, etc.

La mayor parte de los novelistas vieron en esta alianza una oportunidad de ampliar el ámbito de intervención moral en la sociedad; no olvidaban destacar su papel pionero, desarrollado a través de un género literario de comprobada eficacia analítica. Efectivamente, a mediados del siglo XIX la novela había entrado en su periodo de máximo esplendor y no había motivos para que los escritores temiesen la rivalidad. La estética realista anunciaba con triunfalismo una nueva fase en la historia literaria moderna, al rechazar el idealismo retórico y el sentimentalismo; en su lugar proponía la objetividad descriptiva, el análisis crítico y frío de la realidad social, lo que suponía la capacidad de contribuir a su transformación.

Todo el discurso de legitimación de la novela realista tendía explícitamente a asociar este género al método científico, añadiéndole al trabajo creativo un valor suplementario. No sorprende así que el paso siguiente fuese para profundizar esta integración, empezando por la designación que los novelistas atribuyeron a la nueva escuela, el Naturalismo: «La nueva musa es la ciencia experimental de los fenómenos», escribía Eça de Queirós en 1879, en un texto doctrinal, en el que añade:

\footnotetext{
${ }^{4}$ Este desarrollo ha sido estudiado por Wolf Lepenies, Les Trois Cultures. Entre Science et Littérature l'Avènement de la Sociologie (1990).
} 


\begin{abstract}
El naturalismo es la forma científica que adopta el arte, como la república es la forma política que adopta la democracia, como el positivismo es la forma experimental que adopta la filosofía. Todo esto depende y se reduce a esta fórmula general: que fuera de la observación y de la experiencia de los fenómenos, el espíritu no puede obtener ninguna suma de verdad (Eça de Queiros, s. d.: 908 y 914). ${ }^{5}$
\end{abstract}

A partir de los años 1870 se hizo habitual incorporar al discurso literario elementos procedentes de las ciencias «duras» de la época, principalmente las ciencias médicas y biofisiológicas. Además de un imaginario atractivo — la enfermedad (física, moral, social) - la novela recibió de la Medicina coeva toda una topología obsesiva, como la herencia, la histeria o la degeneración. Otra idea motivadora fue el método experimental, etiqueta adaptada por Zola, teniendo como base una obra del fisiólogo Claude Bernard, para promover lo que llamó «novela experimental». Partiendo del conceptoclave de determinismo, ya popularizado en los estudios sociales para explicar al hombre como producto del medio y de su circunstancia, fácil fue deslizarse hacia el ámbito de la biofisiología. La principal originalidad de la novela naturalista consistió precisamente en estudiar los caracteres humanos en función del temperamento y de la sangre o, como diríamos hoy, de su equilibrio genético.

La revolución darwiniana, que tan hondo y perturbador impacto tuvo en la cosmovisión del XIX, no podía dejar de proyectarse también en los textos literarios. Transferidas seguidamente para la fenomenología de las relaciones humanas, las teorías de la evolución y de la selección natural constituyeron temas obligatorios de la literatura de esta época; el darwinismo social inspiró en particular a la narración, que encontró en el struggle for life una metáfora cautivadora para traducir el conflicto novelesco.

Si me he detenido en torno a esta problemática, es porque el Naturalismo representó el episodio más expresivo de la aproximación entre Literatura y Ciencia en nuestra historia moderna. No voy a hacer un análisis más extenso, por haberlo hecho ya en otro trabajo (Santana, 2007). Como entonces afirmé, no son necesariamente positivas las deducciones que se obtienen de este encuentro entusiasta: una de las consecuencias fue la subalternización, en cierta medida autoinfligida, de la práctica literaria al ethos más vanguardista de la ciencia positivista; otra, derivada de esta, sería la clara separación entre novelistas y poetas, temporal, es cierto, pero debilitante para una institución en esos momentos muy influyente y prestigiada.

2. Esta fractura interna, ya latente a lo largo del siglo XIX, se intensificaría al emerger el Simbolismo, principal aglutinador de los muchos «ismos» en que se pulveriza la poesía de finales del siglo. Al contrario de los novelistas, cuya estrategia consistió, como hemos visto, en abrirse hacia temas ideológicos y científicos con repercusión en la sociedad, los poetas del final del siglo prefirieron cultivar el «arte puro», lejos de los valores sociales que consideraban incompatibles con los altos ideales artísticos de la Poesia. A los dogmas del Positivismo - la racionalidad científica y el progreso- oponen el subjetivismo, el misticismo, el esoterismo, en una actitud asumidamente elitista

\footnotetext{
${ }^{5}$ Este texto, escrito para servir de prefacio a la segunda edición de $O$ Crime do padre Amaro, quedó en parte inédito.
} 
de la práctica literaria ${ }^{6}$. En el límite, hasta la referencialidad del lenguaje es puesta en causa, al comunicar la poesía con los happy few, a través de um discurso metafórico extraordinariamente codificado — «para los raros sólo», en palabras del portugués Eugénio de Castro-.

Debe notarse que la reacción de los «poetas nuevos» no se limitó a dirigirse contra sus iguales: en el centro de la disidencia hay también una idea restrictiva, anti-burguesa, sobre el sitio de la Literatura en el espacio sociocultural circundante. Me refiero, concretamente, al «conflicto de las modernidades», expresión consagrada por Matei Calinescu al analizar la tensión cultural que acompaña al triunfo de la civilización burguesa ocidental ${ }^{7}$. Según Calinescu, este fenómeno reactivo se fue consolidando de forma difusa pero irreversible a través de las sucesivas generaciones literarias pos-románticas: se afirma así una conciencia estética moderna globalmente hostil a todos los valores inherentes a la modernidad civilizacional — «la doctrina del progreso, la confianza en las posibilidades benéficas de la ciencia y de la tecnología, la preocupación por el tiempo [...] el culto de la razón [...], de la acción y del éxito» (1987: 49).

La llegada del Modernismo podría hacernos pensar que el malestar de los artistas (o la autoalienación, si lo preferimos) se disiparía por sí mismo, no pasando de un epifenómeno de la época. Efectivamente, una de las principales fuentes de inspiración de la estética modernista fue, como sabemos, la vibración mercantil y tecnológica de la civilização industrial, epitomizada en la gran metrópoli urbana. Y una de las corrientes más conspicuas de este periodo, el futurismo, llegó a elegir a la tecnología mecánica (la fábrica, la máquina, el motor) como objeto de celebración de una nueva e inesperada idea de belleza. Así lo proclama Álvaro de Campos, en los primeros versos de la Oda Triunfal (Pessoa, 1944: 144).

\footnotetext{
A la dolorosa luz de las grandes bombillas de la fábrica tengo fiebre y escribo.

Escribo con crujir de dientes, fiera para la belleza de esto, para la belleza de esto totalmente desconocida para los antigos.
}

Nótese, sin embargo, que no se exalta la ciencia per se, y mucho menos sus virtualidades sociales, que le son totalmente indiferentes al poeta futurista: lo que se exalta, como observa también Calinescu, es el mito de la ciencia, «debido a su potencial metafórico antiartístico y antihumanista» (1987: 119). En realidad, más que mito, hay sobre todo una mística de la tecnología — «Nueva Revelación metálica y dinámica de Dios!», como se lee en la Oda Triunfal (Pessoa, 1944: 149)— que se superpone a la tradición humanista de la cultura occidental. Más allá del imaginario provocador y de la plasticidad formal, el tecnicismo invadió el área «espiritual» de los valores y se erigió en categoría cultural. Esto mismo fue lo que constató Renato Poggioli en su clásico estudio sobre la Vanguardia, al subrayar una visión del mundo marcada por el concepto, tan científico como metafísico, de energía: «This means

\footnotetext{
${ }^{6}$ Pierre Bourdieu, al analizar detalladamente el estado de las artes a finales del siglo XIX, destaca esta ruptura que tiene lugar en el interior del campo literario, basándose en la oposición entre los géneros: por un lado la poesía, en la cima de la jerarquía simbólica, por otro, la novela, en claro ascenso pero aún conotada por el mercantilismo y la vulgaridad (Bourdieu, 1992: 139-47).

7 «Lo que define a la Modernidad cultural es su tajante rechazo de la Modernidad burguesa, su consumidora pasión negativa» (Calinescu, 1987: 49).
} 
that avant-garde scientificism is the particular expression not only of the cult of technique, but also of the general dynamism which is one of the idols of modern culture and was elaborated into a cosmic myth by romantic philosophers» (Poggioli, 1968: 139).

En el mismo espíritu de «dinamismo cósmico» vemos surgir, junto a la técnica, un creciente entusiasmo en la poesia moderna (en el Surrealismo, especialmente) por materias tan variadas como el electromagnetismo, la termodinámica, la teoría de la relatividad, o incluso la geometría o la numerología algebraica, que surgen ostensivamente en los títulos de los libros y de los manifiestos de los muchos «ismos» concurrentes. Véanse, como ejemplo, los manifiestos inter-artísticos del «Dimensionismo»y del «Planismo», suscritos y difundidos en Portugal por António Pedro, en los años treinta, cuyo fundamento espacial apela, sincréticamente, a la teoría de la relatividad, a la geometría pos-euclidiana y a la transmisión óptica (Pedro, 1998: 93-107).

Pero el culto del genio científico estaba fatalmente condenado a la erosión (o a la vulgarización burguesa, como también resalta Poggioli), por la propia lógica auto-destructiva de la vanguardia. Hechos los manifiestos y los himnos, pasada la fase eufórica, el relativismo individualista se superpone, bajo la tónica del humor o de la melancolía. La indiferencia nihilista aparece, por otra parte, muy pronto en los versos spleenáticos del mismo Álvaro de Campos, «un técnico [...] sólo dentro de la técnica»:

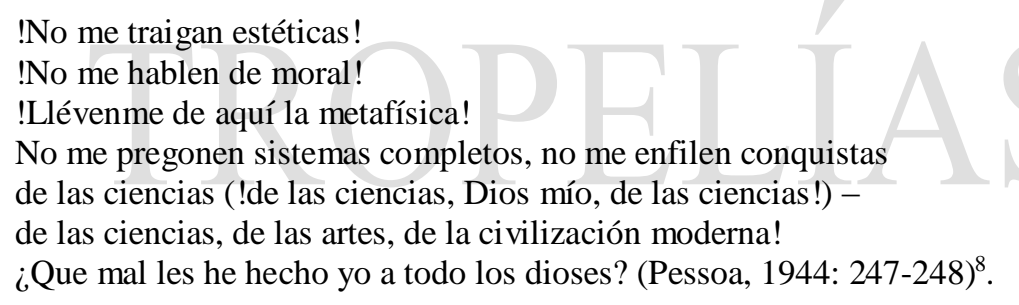

3. El humor es la otra respuesta posible. Se acentúa sobre todo a partir del Surrealismo, en que ya se observa una actitud irónica que subvierte el metaforismo científico, y no dejará de contaminar a la llamada «poesía experimental» de los años sesenta y setenta. El hecho de que esta integre en su semiosis pan-artística una explícita inspiración científica, recurriendo a su lenguaje simbólico-visual, por ejemplo, tiene muchas veces el irónico efecto de reducir ambas (poesía y ciencia) a puro juego formal, divertido e intrínsecamente absurdo ${ }^{9}$. También la poesía puede ser una técnica, pero capaz de autocriticarse, en una infinita parodia de los discursos que nos rigen.

Pero será principalmente en el Pos-Modernismo - y, dentro de este, en el modo fíccionaldonde la actitud humorística adquiere consistencia crítica, condicionando de forma radical las relaciones entre la Literatura y la Ciencia. En este caso, nótese, la ruptura no partió de un deliberado

\footnotetext{
${ }^{8}$ Álvaro de Campos, «Lisbon revisited» (1923).

${ }^{9}$ Cf. E. M. de Melo e Castro, «Soneto Soma 14x», constituido por catorce versos hechos de secuencias numéricas cuya suma totaliza catorce, seguido de un último verso que suma veintiocho. Del mismo autor, el poema «Requiem entropiano» forma un juego visual-verbal que evoluciona desde las raíces tecno- (tecnocrata), etno- y entro- hacia sus múltiples derivados creativos, terminando en tecnotruque, etnonunca, entromorres... Véase Sousa y Ribeiro (2004: 117 y 196).
} 
ensimismamiento del arte frente a la cultura burguesa dominante, como sucedió en las vanguardias modernistas (muy al contrario), sino del cambio de paradigma que se produjo en la misma esfera cultural. Como sabemos, la conciencia pos-moderna nace de un escepticismo epistemológico generalizado — «il pensiero debole», según la obra homónima de Vattimo y Rovatti- que admite diferentes lecturas. En la versión más pesimista se trata de la consumación previsible del declive de una era (el «fin» de la Historia, de la ontología o de la razón científica); en la optimista, de apertura hacia una nueva episteme, tan dispersiva como creativa. Esta última, predominante, descansa en las ideas de falta de certeza y de indeterminación, que son, en parte, como comenta Ihab Hassan, derivaciones de la Física teórica del siglo XX: «In brief, relativity, complementarity, and incompleteness are not simply mathematical idealizations; they are concepts that begin to constitute our cultural languages; they are part of a new order of knowledge founded on both indeterminacy and immanence. In them, we witness signal examples of the "dispersal of discourse"» (Hassan, 1980: $105)^{10}$.

Se popularizó, así, una línea de pensamiento que opone al conocimiento acumulativo y universalista un enfoque pluri-interpretativo del mundo y del hombre; al saber transmitido, certificado por el determinismo científico, le sucede una actitud dubitativa e indagadora, por definición provisional; y en lugar de las metanarrativas culturales, de sentido finalístico, se proponen innumerables perspectivas atomizadas (míticas, inclusive), con derecho al estatuto de conocimiento alternativo.

Muy atacado por el relativismo contracultural que instituye y por la ligereza crítica de sus aporías, el Pos-Modernismo fue naturalmente bien acogido en los sectores de la cultura más marginados por la tradición ilustrada. La Literatura conquistó en este contexto una renovada atención y un consecuente impulso creativo, ya que en gran medida le proporcionó argumentos al debate críticofilosófico. Uno de los aspectos recurrentes de la ficción pos-moderna es justamente el diálogo que establece con la Ciencia como institución universal, productora de una cosmovisión uniforme e impositiva. Al privilegiar miradas «inocentes» - propias de las culturas subdesarrolladas o premodernas, por ejemplo-, las narrativas contemporáneas tienen por costumbre subvertir la lógica racional, representando formas intuitivas de interpretación. De García Márquez a Salman Rushdie o a José Saramago, muchos son los autores que podríamos invocar para ilustrar esta tendencia: hombro a hombro con la ideología política, la magia y el mito irrumpen en la novela, desalojando a la razón y produciendo un reencanto imaginativo, algo utópico, del mundo.

Una derivación más sofisticada se observa en el cuento contemporáneo, que mucho le debe a la imaginación desestabilizadora de Borges y Cortázar. Simplificando, diríamos que se trata de concebir lo real -o las reglas que rigen nuestra percepción de él- como un producto falaz de la racionalidad. Por el contrario, la realidad presenta contornos difusos, caóticos, y no se divisa la lógica que los pueda encuadrar en un orden coherente. Lo insólito es regla. El colapso de los sistemas y de los instrumentos

\footnotetext{
${ }^{10}$ Las teorías de la Relatividad, de la Incertidumbre o del Caos han inspirado algo eufóricamente el pensamiento teóricoliterario y filosófico sobre la Pos-Modernidad.
} 
de regulación - del tiempo, de la vida, de la comunicación...- se convirtió desde entonces en una de las temáticas más productivas del cuento fantástico, género propicio a la deconstrucción del saber. Y las leyes científicas, formuladas para explicar la regularidad del mundo empírico, por ser falibles constituyen un objeto preferencial de irrisión ${ }^{11}$.

Ninguno de estos temas es en sí mismo innovador. No olvidemos que aún antes del paradigma pos-moderno la ficción no-realista venía registrando una actitud ambivalente ante la Ciencia — de fascinación, ansiedad y miedo, a veces simultáneamente-, sobre todo en lo que respecta a la vertiente tecnológica. Frecuentemente el cuento fantástico de inspiración científica (Poe, Hoffmann, Gomes Leal, vg.) presentaba los fenómenos oscuros que se adivinaba en el rastro de las inquietantes revelaciones de las ciencias, o que se resisten a una explicación (la telepatía, el magnetismo cósmico, la combustión espontánea...).

Por otro lado, muchas novelas del futuro ( «anticipation novels») que anticipan la moderna ficción científica tienen tanto de utopía como de distopía: pensemos en obras influyentes como The Time Machine, de H. G. Wells, L'Eve Future, de Villiers de l'Isle-Adam, o, ya en el siglo Xx, Brave New World, de Aldous Huxley, y 1984, de George Orwell; los ejemplos portugueses son escasos, pero se podrá mencionar La ciudad y las sierras, de Eça de Queirós, y El famoso Galrão, de Teixeira de Queirós. En todas ellas la perspectiva de un «extraordinario mundo nuevo», que ya se adivina dominado por la mecanización y por las telecomunicaciones, no esconde el miedo al futuro, el despertar de fuerzas indomables o el fantasma herético de la deshumanización. Por lo demás, los mismos fantasmas (esos demónios despertados por Fausto) ensombrecen aún hoy la ficción científica que, actualizando el imaginario negro, nos propone universos paralelos casi siempre salvajes, poblados de ovnis, aliens o cyborgs.

La originalidad pos-moderna no descansa, por consiguiente, en el aspecto temático, sino en la dimensión crítica y/o paródica. A través del humor y de la ironía se cuestiona la Ciencia pero especialmente los discursos llamados «autoritarios» o «arrogantes» que son sus portadores. En suma: si en la ficción futurística la fantasía científica procura sobre todo conjurar el miedo, en el texto posmoderno está al servicio de la refutación.

Para concluir esta breve reflexión me gustaría regresar al postulado de Jacques Dubois citado en la introducción: los sistemas de pensamiento (filosóficos, científicos, políticos...) se manifiestam oblicuamente en las prácticas literarias; frente a estos, la Literatura se coloca en posición de reserva, o de suplemento. Los términos usados por el autor — «réserve», «suppléance»— no son exactamente sinónimos: según los interpretemos en su acepción débil o fuerte, remiten al sentido de compensación o al sentido de sustitución, pero ambos presuponen, en lo que respecta a los paradigmas dominantes,

\footnotetext{
11 Algunos cuentos de Mário de Carvalho podrían señalarse como ejemplos. Uno de los más sugestivos es «El nudo estadístico» (en La inaudita guerra de la Avenida Gago Coutinho y otras historias, 1992): una pareja de intelectuales se ve ante el insólito fenómeno de un mono que escribe partes de Menina e Moça al ordenador; la consulta a los diferentes sabios expone al absurdo el lenguaje de las ciencias al intentar explicar lo inexplicable.
} 
una idea de incompletudine, de insuficiencia. Dicho de outra manera, la escritura literaria se constituye modernamente como sistema alternativo de un espacio de resistencia a la hegemonía.

De acuerdo con este punto de vista, he procurado rastrear en la historia literaria reciente algunas manifestaciones sensibles del diálogo entre dos de las más notables instituciones humanas —la Literatura y la Ciencia- subrayando la tensión que le es inherente. Correspondiendo a los tres momentos analizados, hemos podido observar las formas algo contradictorias en que esa tensión se expresa, por parte de la Literatura, no en relación al saber científico en sí mismo, sino a su aura simbólica: mimetismo, mitificación o refutación, son las respuestas posibles -o tal vez oblicuas- a una cultura hegemónica que, al sobrestimar el pragmatismo, remite la creación artística a una condición, digamos, residual ${ }^{12}$.

Si pensamos en la bipolaridad Ciencias-Humanidades, una matriz dialéctica de nuestra cultura moderna, el sentido agonístico se hace más perceptible. Al proponer a la Ciencia como representación «inteligible», integral e infinitamente perfeccionada del mundo, la cultura pos-ilustrada creó dentro de sí misma una dimensión conflictual. Lo llamemos «desencanto», en lenguaje weberiano, o «conflicto de las modernidades», el pathos se reconfigura pero se mantiene esencialmente el mismo fundamento: la necesidad de reaccionar a una visión reductora, por ser uniforme, del universo empírico, de la vida y del ser. Por su dimensión simbólico-cognitiva, la Literatura desempeña, en el plano global de la cultura, un papel inalienable. Podríamos invocar el carácter identitario que G. Vattimo le atribuye a la creación artística, en el sentido en que sólo esta le permite a un grupo social, en un determinado momento histórico, reconocer «los rasgos constitutivos de su propia experiencia del mundo, [...] los criterios secretos de distinción entre verdadero y falso, bien y mal» (Vattimo, 1985: 53); nos bastará un argumento más trivial: las artes nos hacen conscientes de que en todas las culturas hay diferentes formas de mirar y de situarse en el mundo, diversos lenguajes para interpretar lo real.

\section{Referencias bibliográficas}

Aguiar e Silva, Vítor M. (1967): Teoria da Literatura. Coimbra, Almedina, 1984, 6 a ed. revisada. BÉnichou, Paul (1977): Le Temps des Prophètes. Doctrines de l'Âge Romantique. Paris, Gallimard. BOURDIEU, Pierre (1992): As Regras da Arte. Lisboa, Presença, 1996.

CAlinescu, Matei (1987): As Cinco Faces da Modernidade. Lisboa, Vega, 1999.

Carvalho, Mário de (1992): A Inaudita Guerra da Avenida Gago Coutinho e Outras Histórias. Lisboa, Caminho.

CHARLE, Christophe (1990): Naissance des Intellectuels (1880-1890). Paris, Minuit. DuboIS, Jacques (1986): L'Institution de la Littérature. Paris, Labor-F. Nathan. EÇA DE QuEIRós, José Maria (s. d): «Idealismo e Realismo», en Obras Completas de Eça de Queiroz. Porto, Lello \& Irmão Editores, vol. III.

\footnotetext{
12 Véanse las consideraciones de Gianni Vattimo sobre el «ocaso del arte», con su disolución en la cultura de masas o el cierre en el silencio, en O Fim da Modernidade (1985, cap. III).
} 
128 Tropelías. Revista de Teoría de la Literatura y Literatura Comparada, 25 (2016)

Maria Helena Santana

Hassan, Ihab (1980): The Right Promethean Fire. Imagination, Science and Cultural Change. Champaign, University of Illinois Press.

LEPENIES, Wolf (1990): Les Trois Cultures. Entre Science et Littérature l'Avènement de la Sociologie. Paris, Maison des Sciences de l'Homme.

Midgley, Mary (1992): Science as Salvation. A Modern Myth and Its Meaning. London-New York, Routledge.

Pedro, António (1998): Antologia Poética. Fernando Matos Oliveira, ed. Braga-Coimbra, Angelus Novus.

PessoA, Fernando (1944): Poesias de Álvaro de Campos. Lisboa, Ática, 1986.

Poggioli, Renato (1968): «Technology and the Avant-Garde», en The Theory of the Avant-Garde. Cambridge (MA)-London, Harvard University Press.

Polanco, Xavier, dir. (1989): Naissance et Dévelopement de la Science-Monde. Paris-Strasbourg, La Découverte-Conseil de L'Europe-Unesco.

Santana, M. Helena (2007): Literatura e Ciência na Ficção do Século XIX. A Narrativa Naturalista e Pós-naturalista Portuguesa. Lisboa, Imprensa Nacional-Casa da Moeda.

SousA, Carlos M. de, y RIBEIRO, Eunice, orgs. (2004): Antologia da Poesia Experimental Portuguesa (Anos 60-Anos 80). Coimbra, Angelus Novus.

Vattimo, Gianni (1985): O Fim da Modernidade. Niilismo e Hermenêutica na Cultura Pós-moderna.

Lisboa, Presença, 1987. 